\title{
An improved pressure regularity criterion of magnetohydrodynamic equations in critical Besov spaces
}

\section{Bo Wang ${ }^{*}$ and Min Chen}

"Correspondence: xnwangbo@163.com School of Mathematics and Statistics, Hubei University of Science and Technology, Xianning, 437100, P.R. China

\begin{abstract}
This paper is concerned with an improved pressure regularity criterion of the three-dimensional (3D) magnetohydrodynamic (MHD) equations in the largest critical Besov spaces. Based on the Littlewood-Paley decomposition technique, the weak solutions are proved to be smooth if the pressure lies in the largest critical Besov spaces, $\pi(x, t) \in L^{p}\left(0, T ; \dot{B}_{q, r}^{0}\left(\mathbf{R}^{3}\right)\right)$ for $\frac{2}{p}+\frac{3}{q}=2,1 \leq r \leq \frac{2 q}{3}, \frac{3}{2}<q \leq \infty$.
\end{abstract}

MSC: $35 \mathrm{Q} 35 ; 76 \mathrm{D} 05$

Keywords: MHD equations; pressure regularity criteria; critical Besov spaces

\section{Introduction and main results}

In this paper, we consider the regularity criterion of the Cauchy problem to the threedimensional (3D) magnetohydrodynamic (MHD) equations in $\mathbf{R}^{3} \times(0, T)$,

$$
\left\{\begin{array}{l}
\partial_{t} v+v \cdot \nabla v-b \cdot \nabla b+\nabla \pi=\gamma \Delta v \\
\partial_{t} b+v \cdot \nabla b-b \cdot \nabla v=\eta \Delta b, \\
\nabla \cdot v=0, \quad \nabla \cdot b=0
\end{array}\right.
$$

associated with the initial condition

$$
v(x, 0)=v_{0}, \quad b(x, 0)=b_{0} .
$$

Here $v(x, t), b(x, t), \pi(x, t)$ are the unknown velocity field, magnetic field and pressure scalar field, respectively. $\gamma \geq 0$ is the kinematic viscosity, $\eta \geq 0$ is the magnetic diffusivity. In particular, when $\gamma=\eta=0$, (1.1) reduces to the ideal MHD equations.

Due to its importance in mathematics, there is a large literature on the well-posedness of the MHD equations [1]. Like the classic Navier-Stokes equations, however, the questions of the global regularity or the finite time singularity for weak solutions of the MHD equations are still big open problems. Therefore, many efforts have been made on the regularity criteria of weak solutions. Caflish et al. [2] showed that if smooth data for the ideal MHD equations leads to a singularity at finite time $T^{*}$, then

$$
\int_{0}^{T^{*}}\left(\|\nabla \times v\|_{L^{\infty}}+\|\nabla \times b\|_{L^{\infty}}\right) d t=\infty .
$$

O 2015 Wang and Chen; licensee Springer. This article is distributed under the terms of the Creative Commons Attribution 4.0 International License (http://creativecommons.org/licenses/by/4.0/), which permits unrestricted use, distribution, and reproduction in any medium, provided you give appropriate credit to the original author(s) and the source, provide a link to the Creative Commons license, and indicate if changes were made. 
As stated by [3], some numerical simulations and observations of space and laboratory plasmas alike reveal that the magnetic field should have some dissipation. Therefore, it is possible to verify that the velocity field plays a more important role than the magnetic field in the regularity theory of solutions to the MHD equations. He and Xin [4] actually examined this observation. Indeed, they proved the regularity of weak solutions of the MHD equations (1.1) if the velocity field satisfies the Serrin condition in critical Lebesgue spaces,

$$
u \in L^{p}\left(0, T ; L^{q}\left(\mathbf{R}^{3}\right)\right) \quad \text { for } \frac{2}{p}+\frac{3}{q} \leq 1,3<q \leq \infty .
$$

The velocity regularity criterion (1.4) was further improved by many authors [5-9]. For example, applying the Fourier localized technique, Chen et al. $[10,11]$ recently refined the regularity criterion in the largest Besov spaces,

$$
u \in L^{p}\left(0, T ; B_{q, \infty}^{s}\left(\mathbf{R}^{3}\right)\right) \quad \text { for } \frac{2}{p}+\frac{3}{q}=1+s, \frac{3}{1+s}<q \leq \infty,-1<s \leq 1 .
$$

At the same time, it is important and interesting to investigate the regularity of weak solutions of the MHD equations (1.1) by imposing some growth conditions on the pressure. Zhou [12] first obtained the result that the weak solutions are smooth if both the pressure $\pi$ and the magnetic field $b$ belong to the Serrin class in the critical Lebesgue spaces

$$
\pi \in L^{p}\left(0, T ; L^{q}\left(\mathbf{R}^{3}\right)\right), \quad b \in L^{2 p}\left(0, T ; L^{2 q}\left(\mathbf{R}^{3}\right)\right) \quad \text { for } \frac{2}{p}+\frac{3}{q} \leq 2, \frac{3}{2}<p \leq \infty .
$$

Recently, Duan [13] removed the restriction on the magnetic field $b$ in (1.6), which implies the magnetic field actually has some dissipation. Cao and Wu [14] considered the regularity of weak solutions in terms of the one component of the gradient of the pressure

$$
\partial_{3} \pi \in L^{p}\left(0, T ; L^{q}\left(\mathbf{R}^{3}\right)\right) \quad \text { for } \frac{2}{p}+\frac{3}{q}=\frac{7}{4}, \frac{12}{7}<q \leq \infty .
$$

Jia and Zhou [15] further improved the pressure regularity criterion

$$
\partial_{3} \pi \in L^{p}\left(0, T ; L^{q}\left(\mathbf{R}^{3}\right)\right) \quad \text { for } \frac{2}{p}+\frac{3}{q} \leq 2, \frac{3}{2}<q \leq \infty .
$$

It should be mentioned that the spaces in (1.7) and (1.8) are subcritical spaces, although the price to pay is the limit for the gradient of the pressure to $\partial_{3} \pi$.

Since the MHD equations become the classic Navier-Stokes equations when the magnetic field $b=0$, one may also refer to some interesting results on pressure regularity criteria of the classic Navier-Stokes equations [16-18] and related fluid models [19, 20]. In particular, Fan et al. [21] (see also Zhang et al. [22], Chen and Zhang [23], and Dong and Chen [24]) proved the pressure regularity criterion of the Navier-Stokes equations in the homogeneous Besov space (see the definition in the next section)

$$
\pi \in L^{1}\left(0, T ; \dot{B}_{\infty, \infty}^{0}\left(\mathbf{R}^{3}\right)\right)
$$


To the best of our knowledge, however, few results have been obtained on the pressure regularity criterion of the MHD in the largest critical spaces. Motivated by the previous regularity criteria results on the MHD and Navier-Stokes equations, the main purpose of this paper is to investigate the pressure regularity criteria of the 3D MHD equations in the largest critical Besov spaces without any additional assumption on the magnetic field $b$. To carry out this aim, we first convert the MHD equations (1.1) into a symmetric form. For convenience, assume $\gamma=\eta=1$ and let $w^{+}=v+b, w^{-}=v-b$. Adding and subtracting (1.1) and (1.2) gives the following equivalent form (see He and Xin [4] for details):

$$
\left\{\begin{array}{l}
\partial_{t} w^{+}+w^{-} \cdot \nabla w^{+}+\nabla \pi=\Delta w^{+}, \\
\partial_{t} w^{-}+w^{+} \cdot \nabla w^{-}+\nabla \pi=\Delta w^{-}, \\
\nabla \cdot w^{+}=0, \quad \nabla \cdot w^{-}=0,
\end{array}\right.
$$

associated with the initial condition

$$
w_{0}^{+}=v_{0}+b_{0}, \quad w_{0}^{-}=v_{0}-b_{0} .
$$

To state our main results, we recall the definitions of weak solutions of the 3D MHD equations (1.1)-(1.2) and their equivalent equations (1.10)-(1.11).

Definition 1.1 (Sermange and Temam [1]) Given $w_{0}^{+}, w_{0}^{-} \in L^{2}\left(\mathbf{R}^{3}\right)$, a pair $\left(w^{+}, w^{-}\right)$on $\mathbf{R}^{3} \times$ $(0, T)$ is called a weak solution of the 3D MHD equations (1.10)-(1.11), provided

(1) $\left(w^{+}, w^{-}\right) \in L^{\infty}\left(0, T ; L^{2}\left(\mathbf{R}^{3}\right)\right) \cap L^{2}\left(0, T ; H^{1}\left(\mathbf{R}^{3}\right)\right), \forall T>0$,

(2) $\nabla \cdot w^{+}=0, \nabla \cdot w^{-}=0$ in the sense of distributions,

(3) for any $\phi, \psi \in C_{0}^{\infty}\left(\mathbf{R}^{3} \times[0, T)\right)$ with $\nabla \cdot \phi=0$ and $\nabla \cdot \psi=0$,

$$
\int_{0}^{T} \int_{\mathbf{R}^{3}}\left\{w^{+} \cdot \partial_{t} \phi-\nabla w^{+} \cdot \nabla \phi+\nabla \phi:\left(w^{-} \otimes w^{+}\right)\right\} d x d t=-\left(w_{0}^{+}, \phi(0)\right)
$$

and

$$
\int_{0}^{T} \int_{\mathbf{R}^{3}}\left\{w^{-} \cdot \partial_{t} \psi-\nabla w^{-} \cdot \nabla \psi+\nabla \psi:\left(w^{+} \otimes w^{-}\right)\right\} d x d t=-\left(w_{0}^{-}, \psi(0)\right) .
$$

The following should be mentioned again. According to the definitions of the above weak solutions, (1.1)-(1.2) and (1.10)-(1.11) are equivalent. Especially, if $(v, b)$ is a weak solution of (1.1)-(1.2), then it is easy to see that $\left(w^{+}, w^{-}\right)$also verifies (1.10)-(1.11) in the sense of distributions.

Now our results read as follows.

Theorem 1.1 Suppose $\left(v_{0}, b_{0}\right) \in L^{2}\left(\mathbf{R}^{3}\right) \cap L^{4}\left(\mathbf{R}^{3}\right)$ and $(v(x, t), b(x, t))$ is a weak solution of the MHD equations (1.1)-(1.2) on $(0, T)$. If the pressure $\pi(x, t)$ satisfies

$$
\pi(x, t) \in L^{p}\left(0, T ; \dot{B}_{q, r}^{0}\left(\mathbf{R}^{3}\right)\right) \quad \text { for } \frac{2}{p}+\frac{3}{q}=2,1 \leq r \leq \frac{2 q}{3}, \frac{3}{2}<q \leq \infty,
$$


Remark 1.1 Since $L^{q} \hookrightarrow \dot{B}_{q, \infty}^{0}$, our result obviously covers the previous ones, (1.6) and (1.9). Indeed, the space-time space in (1.12) on pressure is a degree -2 homogeneous space (see Chen and Xin [25], Chen and Price [26]) and the largest critical space with respect to the previous pressure regularity criteria of the MHD equations.

Remark 1.2 The proof of Theorem 1.1 is mainly based on the Fourier localized methods which are employed where the pressure is decomposed into three parts: low frequency, middle frequency, and high frequency.

Remark 1.3 We do not know whether the pressure regularity criteria (1.7) or (1.8) can be improved to the Besov spaces; that is to say, whether our results are still valid when the critical growth condition (1.12) is limited to $\partial_{3} \pi$. Compared with the previous results, the main difficulty lies in the lack of the anisotropic Sobolev inequalities in the framework of Besov spaces. We will focus on this challenging problem in the future.

\section{Preliminary}

Throughout this paper, $C$ stands for a generic positive constant which may vary from line to line. $L^{p}\left(\mathbf{R}^{3}\right)$ with $1 \leq p \leq \infty$ denotes the usual Lebesgue space of all $L^{p}$ integral functions associated with the norm

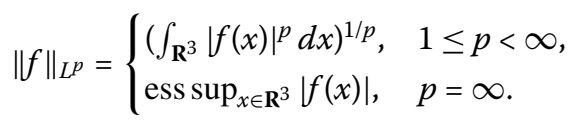

We denote by $\mathcal{S}\left(\mathbf{R}^{3}\right)$ the Schwartz class of rapidly decreasing functions. Given $f \in \mathcal{S}\left(\mathbf{R}^{3}\right)$, its Fourier transformation $\mathcal{F}$ or $\hat{f}$ is defined by

$$
\mathcal{F} f(\xi)=\hat{f}(\xi)=\int_{\mathbf{R}^{3}} e^{-i x \cdot \xi} f(x) d x .
$$

As stated in Remark 1.2, the proof of our result is largely based on the Fourier localized methods. Therefore, it is necessary to introduce the Littlewood-Paley decomposition theory (see Chemin [27]). Choose two nonnegative radial functions $\chi, \varphi \in \mathcal{S}\left(\mathbf{R}^{3}\right)$ supported in $\mathcal{B}=\left\{\xi \in \mathbf{R}^{3}:|\xi| \leq 4 / 3\right\}$ and $\mathcal{C}=\left\{\xi \in \mathbf{R}^{3}: 3 / 4 \leq|\xi| \leq 8 / 3\right\}$, respectively, such that

$$
\sum_{j \in Z} \varphi\left(2^{-j} \xi\right)=1, \quad \xi \in \mathbf{R}^{3} \backslash\{0\}
$$

Thus the Littlewood-Paley projection operator $\Delta_{j}$ is defined by

$$
\Delta_{j} f=\mathcal{F}^{-1} \varphi\left(2^{-j} \xi\right) \mathcal{F} f=\varphi\left(2^{-j} D\right) f
$$

Formally, $\Delta_{j}$ is a frequency projection to the annulus $|\xi| \sim 2^{j}$ and one can easily verify that

$$
\Delta_{j} \Delta_{k} f=0 \quad \text { if }|j-k| \geq 2 .
$$

Especially for any $f \in L^{2}\left(\mathbf{R}^{3}\right)$, we have the Littlewood-Paley decomposition:

$$
f=\sum_{j=-\infty}^{\infty} \Delta_{j} f
$$


Let $s \in \mathbf{R}, q, r \in[1, \infty]$, the homogeneous Besov space $\dot{B}_{q, r}^{s}\left(\mathbf{R}^{3}\right)$ is defined by the fulldyadic decomposition such as

$$
\dot{B}_{q, r}^{s}\left(\mathbf{R}^{3}\right)=\left\{f \in \mathcal{S}^{\prime}\left(\mathbf{R}^{3}\right) / \mathcal{P}\left(\mathbf{R}^{3}\right):\|f\|_{\dot{B}_{q, r}^{s}}<\infty\right\}
$$

where

$$
\|f\|_{\dot{S}_{q, r}^{s}}= \begin{cases}\left(\sum_{j=-\infty}^{\infty} 2^{j s q}\left\|\Delta_{j} f\right\|_{L^{q}}^{r}\right)^{\frac{1}{r}}, & 1 \leq r<\infty \\ \sup _{j \in Z} 2^{j s}\left\|\Delta_{j} f\right\|_{L^{q}}, & r=\infty\end{cases}
$$

and $\mathcal{S}^{\prime}\left(\mathbf{R}^{3}\right), \mathcal{P}\left(\mathbf{R}^{3}\right)$ are the spaces of all tempered distributions on $\mathbf{R}^{3}$ and the set of all scalar polynomials defined on $\mathbf{R}^{3}$, respectively.

In order to prove the main result, we give several important lemmas. Firstly, the following Bernstein inequalities will be frequently used.

Lemma 2.1 (Chemin [27]) For all $k \in N \cup\{0\}, j \in Z$ and $1 \leq p \leq q \leq \infty$, we have for all $f \in \mathcal{S}\left(\mathbf{R}^{3}\right)$,

$$
\sup _{|\alpha|=k}\left\|\nabla^{\alpha} \Delta_{j} f\right\|_{L^{q}} \leq c 2^{j k+3 j(1 / p-1 / q)}\left\|\Delta_{j} f\right\|_{L^{p}}
$$

with $C$ being a positive constant independent of $f, j$.

In order to complete our proofs, we also need the following results on the local smooth solutions and blow up criterion of the 3D MHD equations.

Lemma 2.2 Suppose $\left(w_{0}^{+}, w_{0}^{-}\right) \in L^{2}\left(\mathbf{R}^{3}\right) \cap L^{4}\left(\mathbf{R}^{3}\right)$, then there exist a constant $T>0$ and a unique local strong solution $\left(w^{+}, w^{-}\right)$of (1.10)-(1.11) such that

$$
\left(w^{+}, w^{-}\right) \in B C\left([0, T) ; L^{4}\left(\mathbf{R}^{3}\right)\right) .
$$

Moreover, if $T^{*}$ is the maximal time of existence of the local smooth solution $\left(w^{+}, w^{-}\right)$, then

$$
\left\|\left(w^{+}, w^{-}\right)(s)\right\|_{L^{4}} \geq \frac{C}{\left(T^{*}-s\right)^{1 / 8}} .
$$

Proof of Lemma 2.2 It should be mentioned that for the 3D classic Navier-Stokes equations, the same results have been proved by Giga [28]. The proof of Lemma 2.2 is parallel to that of [28] and thus we omit it for convenience.

\section{A priori estimates on $w^{+}, w^{-}$}

In order to prove the main results, it is necessary to establish a priori estimates for the smooth solutions of the 3D MHD equations. More precisely, we will prove the following theorem.

Theorem 3.1 Suppose $w_{0}^{+}, w_{0}^{-} \in L^{2}\left(\mathbf{R}^{3}\right) \cap L^{4}\left(\mathbf{R}^{3}\right)$ and $\left(w^{+}, w^{-}\right)$is a local smooth solution of (1.10)-(1.11) on $(0, T)$. If the pressure satisfies the critical growth conditions (1.12), then we 
have

$$
\begin{aligned}
& \sup _{0<t<T}\left(\left\|w^{+}\right\|_{L^{4}}^{4}+\left\|w^{-}\right\|_{L^{4}}^{4}\right)+\int_{0}^{T}\left(\left\|w^{+} \nabla w^{+}\right\|_{L^{2}}^{2}+\left\|w^{-} \nabla w^{-}\right\|_{L^{2}}^{2}\right) \\
& \quad \leq C\left(\left\|w_{0}^{+}\right\|_{L^{4}}^{4},\left\|w_{0}^{-}\right\|_{L^{4}}^{4}, T\right) \exp \left\{C \int_{0}^{T}\|\pi(t)\|_{\dot{B}_{q, r}^{0},}^{p} d t\right\} .
\end{aligned}
$$

Proof of Theorem 3.1 We multiply the first equation of (1.10) with $\left|w^{+}\right|^{2} w^{+}$and then, integrating in $\mathbf{R}^{3}$, one shows that

$$
\begin{aligned}
& \frac{1}{4} \frac{d}{d t} \int_{\mathbf{R}^{3}}\left|w^{+}\right|^{4} d x+\int_{\mathbf{R}^{3}}\left|w^{+}\right|^{2}\left|\nabla w^{+}\right|^{2} d x+\left.\left.\frac{1}{2} \int_{\mathbf{R}^{3}}|\nabla| w^{+}\right|^{2}\right|^{2} d x \\
& \quad=-\int_{\mathbf{R}^{3}} w^{+} \cdot \nabla \pi\left|w^{+}\right|^{2} d x,
\end{aligned}
$$

where we have used

$$
\int_{\mathbf{R}^{3}} w^{-} \cdot \nabla w^{+}\left|w^{+}\right|^{2}\left|w^{+}\right|^{2} d x=0
$$

due to the divergence free of $w^{-}$.

Applying the Hölder inequality and the Young inequality, it follows that

$$
\begin{aligned}
& -\int_{\mathbf{R}^{3}} w^{+} \cdot \nabla \pi\left|w^{+}\right|^{2} d x \\
& \quad \leq C \int_{\mathbf{R}^{3}}\left|\pi w^{+}\right|^{2} d x+\frac{1}{8}\left\|\nabla\left|w^{+}\right|^{2}\right\|_{L^{2}}^{2} .
\end{aligned}
$$

By the Littlewood-Paley decomposition introduced in Section 2, we decompose the pressure into three parts: low frequency, middle frequency and high frequency. We have

$$
\begin{aligned}
\int_{\mathbf{R}^{3}}\left|\pi w^{+}\right|^{2} d x & \leq 3 \int_{\mathbf{R}^{3}}\left|\left(\sum_{j<-K}+\sum_{j=-K}^{K}+\sum_{j>K}\right)\left(\Delta_{j} \pi\right)\right||\pi|\left|\left(w^{+}\right)^{2}\right| d x \\
= & : J_{1}+J_{2}+J_{3} .
\end{aligned}
$$

We now estimate them one by one. For $J_{1}$, employing the Hölder inequality and the Bernstein inequality gives

$$
\begin{aligned}
\left|J_{1}\right| & \leq \sum_{j<-K} \int_{\mathbf{R}^{3}}\left|\Delta_{j} \pi \| \pi\right|\left|w^{+}\right|^{2} d x \\
& \leq C \sum_{j<-K}\left\|\Delta_{j} \pi\right\|_{L^{\infty}}\|\pi\|_{L^{2}}\left\|w^{+}\right\|_{L^{4}}^{2} \\
& \leq C \sum_{j<-K} 2^{\frac{3}{2} j}\left\|\Delta_{j} \pi\right\|_{L^{2}}\|\pi\|_{L^{2}}\left\|w^{+}\right\|_{L^{4}}^{2} \\
& \leq C\left(\sum_{j<-K} 2^{3 j}\right)^{\frac{1}{2}}\left(\sum_{j<-K}\left\|\Delta_{j} \pi\right\|_{L^{2}}^{2}\right)^{\frac{1}{2}}\|\pi\|_{L^{2}}\left\|w^{+}\right\|_{L^{4}}^{2}
\end{aligned}
$$




$$
\begin{aligned}
& \leq C 2^{-\frac{3}{2} K}\left\|w^{+}\right\|_{L^{4}}^{2}\left(\left\|w^{+}\right\|_{L^{4}}^{4}+\left\|w^{-}\right\|_{L^{4}}^{4}\right) \\
& \leq C\left(2^{-K}\left(\left\|w^{+}\right\|_{L^{4}}^{4}+\left\|w^{-}\right\|_{L^{4}}^{4}\right)\right)^{\frac{3}{2}}
\end{aligned}
$$

where we have used the fundamental Calderón-Zygmund inequality

$$
\|\pi\|_{L^{2}} \leq C\left\|w^{+} \otimes w^{-}\right\|_{L^{2}} \leq C\left\|w^{+}\right\|_{L^{4}}\left\|w^{-}\right\|_{L^{4}}
$$

due to the integral expression of $\pi$

$$
\pi=(-\Delta)^{-1} \nabla \cdot\left(w^{+} \cdot \nabla w^{-}\right) .
$$

For $J_{2}$, similarly, we have

$$
\begin{aligned}
\left|J_{2}\right| & \leq C \sum_{j=-K}^{K}\left\|\Delta_{j} \pi\right\|_{L^{q}}\|\pi\|_{L^{\frac{2 q}{q-1}}}\left\|w^{+}\right\|_{L^{\frac{4 q}{q-1}}}^{2} \\
& \leq C \sum_{j=-K}^{K}\left\|\Delta_{j} \pi\right\|_{L^{q}}\|\pi\|_{L^{\frac{2 q}{q-1}}\left\|w^{+}\right\|_{L^{\frac{4 q}{q-1}}}^{2}} \\
& \leq C\left(\sum_{j=-K}^{K} 1\right)^{\frac{2 q-3}{2 q}}\left(\sum_{j=-K}^{K}\left\|\Delta_{j} \pi\right\|_{L^{q}}^{\frac{2 q}{9}}\right)^{\frac{3}{2 q}}\|\pi\|_{L^{\frac{2 q}{q-1}}}\left\|w^{+}\right\|_{L^{\frac{4 q}{q-1}}}^{2} \\
& \leq C K^{\frac{2 q-3}{2 q}}\|\pi\|_{\dot{B}_{q, r}^{0}}\|\pi\|_{L^{\frac{2 q}{q-1}}}\left\|w^{+}\right\|_{L^{\frac{4 q}{q-1}}}^{2} \\
& \leq C K^{\frac{2 q-3}{2 q}}\|\pi\|_{\dot{B}_{q, r}^{0}}\left(\left\|w^{+}\right\|_{L^{\frac{4 q}{q-1}}}^{4}+\left\|w^{-}\right\|_{L^{\frac{4 q}{q-}}}^{4}\right),
\end{aligned}
$$

where

$$
1 \leq r \leq \frac{2 q}{3}
$$

Applying the interpolation inequality and the Young inequality, one shows that

$$
\begin{aligned}
& K^{\frac{2 q-3}{2 q}}\|\pi\|_{\dot{B}_{q, r}^{0}}\left(\left\|w^{+}\right\|_{L^{\frac{4 q}{q-1}}}^{4}+\left\|w^{-}\right\|_{L^{\frac{4 q}{q-1}}}^{4}\right) \\
& \leq C K^{\frac{2 q-3}{2 q}}\|\pi\|_{\dot{B}_{q, r}^{0}}\left(\left\|w^{+}\right\|_{L^{4}}^{4-\frac{6}{q}}\left\|w^{+}\right\|_{L^{12}}^{\frac{6}{q}}+\left\|w^{-}\right\|_{L^{4}}^{4-\frac{6}{q}}\left\|w^{-}\right\|_{L^{12}}^{\frac{6}{q}}\right) \\
& \leq C(\varepsilon) K\|\pi\|_{\dot{B}_{q, r}^{0}}^{p}\left(\left\|w^{+}\right\|_{L^{4}}^{4}+\left\|w^{-}\right\|_{L^{4}}^{4}\right)+\varepsilon\left(\left\|\left|w^{+}\right|^{2}\right\|_{L^{6}}^{2}+\left\|\left|w^{-}\right|^{2}\right\|_{L^{6}}^{2}\right) \\
& \leq C(\varepsilon) K\|\pi\|_{\dot{B}_{q, r}^{0}}^{p}\left(\left\|w^{+}\right\|_{L^{4}}^{4}+\left\|w^{-}\right\|_{L^{4}}^{4}\right)+\varepsilon\left(\left\|\nabla\left|w^{+}\right|^{2}\right\|_{L^{2}}^{2}+\left\|\nabla\left|w^{-}\right|^{2}\right\|_{L^{2}}^{2}\right) \\
& \leq C K\|\pi\|_{\dot{B}_{q, r}^{0}}^{p}\left(\left\|w^{+}\right\|_{L^{4}}^{4}+\left\|w^{-}\right\|_{L^{4}}^{4}\right)+\frac{1}{8}\left(\left\|\nabla\left|w^{+}\right|^{2}\right\|_{L^{2}}^{2}+\left\|\nabla\left|w^{-}\right|^{2}\right\|_{L^{2}}^{2}\right),
\end{aligned}
$$

where

$$
p=\frac{2 q}{2 q-3}
$$


Plugging (3.7) into (3.6), we have

$$
\begin{aligned}
\left|J_{2}\right| \leq & C K\|\pi\|_{\dot{B}_{q, r}^{0}}^{p}\left(\left\|w^{+}\right\|_{L^{4}}^{4}+\left\|w^{-}\right\|_{L^{4}}^{4}\right) \\
& +\frac{1}{8}\left(\left\|\nabla\left|w^{+}\right|^{2}\right\|_{L^{2}}^{2}+\left\|\nabla\left|w^{-}\right|^{2}\right\|_{L^{2}}^{2}\right) .
\end{aligned}
$$

For $J_{3}$, with the aid of the Bernstein inequality, the Hölder inequality, and the GagliardoNirenberg inequality, one shows that

$$
\begin{aligned}
J_{3} & \leq C \sum_{j>K}\left\|\Delta_{j} \pi\right\|_{L^{3}}\|\pi\|_{L^{2}}\left\|\left|w^{+}\right|^{2}\right\|_{L^{6}} \\
& \leq C \sum_{j>K} 2^{\frac{j}{2}}\left\|\Delta_{j} \pi\right\|_{2}\left\|w^{+}\right\|_{L^{4}}^{2}\left\|\nabla\left|w^{+}\right|^{2}\right\|_{L^{2}} \\
& \leq C\left(\sum_{j>K} 2^{-j}\right)^{\frac{1}{2}}\left(\sum_{j>K} 2^{2 j}\left\|\Delta_{j} \pi\right\|_{2}^{2}\right)^{\frac{1}{2}}\left\|w^{+}\right\|_{L^{4}}^{2}\left\|\nabla\left|w^{+}\right|^{2}\right\|_{L^{2}} \\
& \leq C 2^{-\frac{K}{2}}\|\pi\|_{\dot{B}_{2,2}^{1}}\left\|w^{+}\right\|_{L^{4}}^{2}\left\|\nabla\left|w^{+}\right|^{2}\right\|_{L^{2}} \\
& \leq C 2^{-\frac{K}{2}}\|\nabla \pi\|_{L^{2}}\left\|w^{+}\right\|_{L^{4}}^{2}\left\|\nabla\left|w^{+}\right|^{2}\right\|_{L^{2}} \\
& \leq C 2^{-\frac{K}{2}}\left(\left\|\nabla\left|w^{+}\right|^{2}\right\| L_{L^{2}}+\left\|\nabla\left|w^{-}\right|^{2}\right\| L_{L^{2}}\right)\left\|w^{+}\right\|_{L^{4}}^{2}\left\|\nabla\left|w^{+}\right|^{2}\right\|_{L^{2}} \\
& \leq C 2^{-\frac{K}{2}}\left\|w^{+}\right\|_{L^{4}}^{2}\left(\left\|\nabla\left|w^{+}\right|^{2}\right\|_{L^{2}}^{2}+\left\|\nabla\left|w^{-}\right|^{2}\right\|_{L^{2}}^{2}\right) \\
& \leq C\left(2^{-K}\left(\left\|w^{+}\right\|_{L^{4}}^{4}+\left\|w^{-}\right\|_{L^{4}}^{4}\right)\right)^{\frac{1}{2}}\left(\left\|\nabla\left|w^{+}\right|^{2}\right\|_{L^{2}}+\left\|\nabla\left|w^{-}\right|^{2}\right\|_{L^{2}}\right) .
\end{aligned}
$$

Plugging the estimates of $J_{1}, J_{2}, J_{3}$ into the inequality (3.3), it follows that

$$
\begin{aligned}
& \frac{d}{d t} \int_{\mathbf{R}^{3}}\left|w^{+}\right|^{4} d x+\left.\left.\frac{1}{2} \int_{\mathbf{R}^{3}}|\nabla| w^{+}\right|^{2}\right|^{2} d x \\
& \leq C\left(2^{-K}\left(\left\|w^{+}\right\|_{L^{4}}^{4}+\left\|w^{-}\right\|_{L^{4}}^{4}\right)\right)^{\frac{3}{2}}+C K\|\pi\|_{\dot{B}_{q, r}^{0}}^{p}\left(\left\|w^{+}\right\|_{L^{4}}^{4}+\left\|w^{-}\right\|_{L^{4}}^{4}\right) \\
& \quad+C\left(2^{-K}\left(\left\|w^{+}\right\|_{L^{4}}^{4}+\left\|w^{-}\right\|_{L^{4}}^{4}\right)\right)^{\frac{1}{2}}\left(\left\|\nabla\left|w^{+}\right|^{2}\right\|_{L^{2}}+\left\|\nabla\left|w^{-}\right|^{2}\right\|_{L^{2}}\right)
\end{aligned}
$$

At the same time, we can obtain a similar inequality to (3.10) for $w^{-}$. We have

$$
\begin{aligned}
\frac{d}{d t} \int_{\mathbf{R}^{3}}\left|w^{-}\right|^{4} d x+\left.\left.\frac{1}{2} \int_{\mathbf{R}^{3}}|\nabla| w^{-}\right|^{2}\right|^{2} d x \\
\leq C\left(2^{-K}\left(\left\|w^{+}\right\|_{L^{4}}^{4}+\left\|w^{-}\right\|_{L^{4}}^{4}\right)\right)^{\frac{3}{2}}+C K\|\pi\|_{\dot{B}_{q, r}^{0}}^{p}\left(\left\|w^{+}\right\|_{L^{4}}^{4}+\left\|w^{-}\right\|_{L^{4}}^{4}\right) \\
\quad+C\left(2^{-K}\left(\left\|w^{+}\right\|_{L^{4}}^{4}+\left\|w^{-}\right\|_{L^{4}}^{4}\right)\right)^{\frac{1}{2}}\left(\left\|\nabla\left|w^{+}\right|^{2}\right\|_{L^{2}}+\left\|\nabla\left|w^{-}\right|^{2}\right\|_{L^{2}}\right) .
\end{aligned}
$$

Choosing $K$ such that

$$
C\left(2^{-K}\left(\left\|w^{+}\right\|_{L^{4}}^{4}+\left\|w^{-}\right\|_{L^{4}}^{4}\right)\right)^{\frac{1}{2}} \leq \frac{1}{4},
$$


i.e. we only need to let $K$

$$
K=\frac{\ln C\left(e+\left\|w^{+}\right\|_{L^{4}}^{4}+\left\|w^{-}\right\|_{L^{4}}^{4}\right)}{\ln 2}+4
$$

then adding (3.10) to (3.11) gives

$$
\begin{aligned}
& \frac{d}{d t} \int_{\mathbf{R}^{3}}\left(\left|w^{+}\right|^{4}+\left|w^{-}\right|^{4}\right) d x+\frac{1}{2} \int_{\mathbf{R}^{3}}\left(\left.\left.|\nabla| w^{+}\right|^{2}\right|^{2}+\left.\left.|\nabla| w^{-}\right|^{2}\right|^{2}\right) d x \\
& \quad \leq C+C K\|\pi\|_{\dot{B}_{q, r}^{0}}^{p}\left(\left\|w^{+}\right\|_{L^{4}}^{4}+\left\|w^{-}\right\|_{L^{4}}^{4}\right) \\
& \quad \leq C+C\left\{\ln \left(e+\left\|w^{+}\right\|_{L^{4}}^{4}+\left\|w^{-}\right\|_{L^{4}}^{4}\right)\right\}\|\pi\|_{\dot{B}_{q, r}^{0}}^{p}\left(\left\|w^{+}\right\|_{L^{4}}^{4}+\left\|w^{-}\right\|_{L^{4}}^{4}\right),
\end{aligned}
$$

and taking the Gronwall inequality into account yields

$$
\begin{aligned}
\left\|w^{+}(t)\right\|_{L^{4}}^{4}+\left\|w^{-}(t)\right\|_{L^{4}}^{4} & \\
\leq & \left(\left\|w_{0}^{+}\right\|_{L^{4}}^{4}+\left\|w_{0}^{-}\right\|_{L^{4}}^{4}+C T\right) \\
\quad & \quad \exp \left\{\int_{0}^{t}\left\{\ln \left(e+\left\|w^{+}\right\|_{L^{4}}^{4}+\left\|w^{-}\right\|_{L^{4}}^{4}\right)+C\right\}\|\pi\|_{\dot{B}_{q, r}^{0}}^{p} d t\right\}
\end{aligned}
$$

or

$$
\begin{aligned}
& \ln \left(\left\|w^{+}(t)\right\|_{L^{4}}^{4}+\left\|w^{-}(t)\right\|_{L^{4}}^{4}+e\right) \\
& \leq \ln \left(\left\|w_{0}^{+}\right\|_{L^{4}}^{4}+\left\|w_{0}^{-}\right\|_{L^{4}}^{4}+C T\right)+C T \\
& \quad+\int_{0}^{t}\left(\ln \left(e+\left\|w^{+}\right\|_{L^{4}}^{4}+\left\|w^{-}\right\|_{L^{4}}^{4}\right)+C\right)\|\pi\|_{\dot{B}_{q, r}^{0}}^{p} d t .
\end{aligned}
$$

Applying the Gronwall inequality again, we obtain the estimates of $w^{+}, w^{-}$

$$
\begin{aligned}
& \ln \left(\left\|w^{+}(t)\right\|_{L^{4}}^{4}+\left\|w^{-}(t)\right\|_{L^{4}}^{4}+e\right) \\
& \quad \leq\left(\ln \left(\left\|w_{0}^{+}\right\|_{L^{4}}^{4}+\left\|w_{0}^{-}\right\|_{L^{4}}^{4}+C T\right)+C T^{2}\right) \exp \left\{C \int_{0}^{T}\|\pi\|_{\dot{B}_{q, r}^{0}}^{p} d t\right\},
\end{aligned}
$$

from which one derives the assertion of (3.1).

\section{Proof of Theorem 1.1}

According to a priori estimates of the smooth solution in Theorem 3.1, now we are in a position to complete the proof of Theorem 1.1, which is more or less standard.

Since $\left(v_{0}, b_{0}\right) \in L^{2}\left(\mathbf{R}^{3}\right) \cap L^{4}\left(\mathbf{R}^{3}\right)$, i.e. $\left(w_{0}^{+}, w_{0}^{-}\right) \in L^{2}\left(\mathbf{R}^{3}\right) \cap L^{4}\left(\mathbf{R}^{3}\right)$, according to Lemma 2.2, there exist a $T^{*}>0$ and a local strong solution $\left(w^{+}, w^{-}\right)$of the MHD equations (1.10)-(1.11) satisfying $\left(w^{+}, w^{-}\right) \in B C\left(\left[0, T^{*}\right) ; L^{4}\left(\mathbf{R}^{3}\right)\right)$. At the same time, according to Theorem 3.1, there also exists a local smooth solution $\left(w_{1}^{+}, w_{1}^{-}\right)$on $(0, T)$ and it satisfies

$$
\left(w^{+}, w^{-}\right) \equiv\left(w_{1}^{+}, w_{1}^{-}\right) \quad \text { on }\left[0, T^{*}\right)
$$

Thus it is sufficient to show that $T^{*} \geq T$. Suppose that $T^{*}<T$. Without loss of generality, we may assume that $T^{*}$ is the maximal existence time for the solution $\left(w^{+}, w^{-}\right)$. Since the 
pressure satisfies (1.12), it follows from the a priori estimates in Theorem 3.1 that the existence time of the solution $\left(w^{+}, w^{-}\right)$can be extended after $t>T^{*}$, which contradicts the maximality of $t=T^{*}$. Thus we proved $\left(w^{+}, w^{-}\right)$is smooth, which implies the solution $(v, b)$ is also smooth.

\section{Competing interests}

The authors declare that they have no competing interests.

\section{Authors' contributions}

All authors contributed equally to the writing of this paper. All authors read and approved the final manuscript.

\section{Acknowledgements}

The authors want to express their sincere thanks to the referees for their invaluable comments and suggestions, which helped to improve the paper greatly.

Received: 15 November 2014 Accepted: 10 April 2015 Published online: 23 April 2015

\section{References}

1. Sermange, $M$, Temam, R: Some mathematical questions related to the MHD equations. Commun. Pure Appl. Math. 36, 635-664 (1983)

2. Caflish, R, Klapper, I, Steele, G: Remarks on singularities,dimension and energy dissipation for ideal hydrodynamics and MHD. Commun. Math. Phys. 184, 443-455 (1997)

3. Politano, H, Pouquet, A, Sulem, PL: Current and vorticity dynamics in three-dimensional magnetohydrodynamics turbulence. Phys. Plasmas 2, 2931-2939 (1995)

4. He, C, Xin, Z: On the regularity of solutions to the magnetohydrodynamic equations. J. Differ. Equ. 213, 235-254 (2005)

5. Dong, B, Jia, Y, Zhang, W: An improved regularity criterion of three-dimensional magnetohydrodynamic equations. Nonlinear Anal., Real World Appl. 13, 1159-1169 (2012)

6. He, C, Wang, Y: On the regularity criteria for weak solutions to the magnetohydrodynamic equations. J. Differ. Equ. 238, 1-17 (2007)

7. Jia, X, Zhou, Y: Regularity criteria for the 3D MHD equations involving partial components. Nonlinear Anal., Real World Appl. 13, 410-418 (2012)

8. Wu, J: Regularity results for weak solutions of the 3D MHD equations. Discrete Contin. Dyn. Syst. 10, 543-556 (2004)

9. Zhou, Y, Gala, S: Regularity criteria for the solutions to the 3D MHD equations in the multiplier space. Z. Angew. Math. Phys. 61, 193-199 (2010)

10. Chen, Q, Miao, C, Zhang, Z: The Beale-Kato-Majda criterion for the 3D magneto-hydrodynamics equations. Commun. Math. Phys. 275, 861-872 (2007)

11. Chen, Q, Miao, C, Zhang, Z: On the regularity criterion of weak solution for the $3 \mathrm{D}$ viscous magneto-hydrodynamics equations. Commun. Math. Phys. 284, 919-930 (2008)

12. Zhou, Y: Regularity criteria for the 3D MHD equations in terms of the pressure. Int. J. Non-Linear Mech. 41, 1174-1180 (2006)

13. Duan, H: On regularity criteria in terms of pressure for the 3D viscous MHD equations. Appl. Anal. 91, 947-952 (2011)

14. Cao, C, Wu, J: Two regularity criteria for the 3D MHD equations. J. Differ. Equ. 248, 2263-2274 (2010)

15. Jia, $X, Z$ Zhou, Y: A new regularity criterion for the $3 D$ incompressible MHD equations in terms of one component of the gradient of pressure. J. Math. Anal. Appl. 396, 345-350 (2012)

16. Dong, B, Zhang, Z: On the weak-strong uniqueness of Koch-Tataru's solution for the Navier-Stokes equations. J. Differ. Equ. 256, 2406-2422 (2014)

17. Berselli, L, Galdi, G: Regularity criteria involving the pressure for the weak solutions to the Navier-Stokes equations. Proc. Am. Math. Soc. 130, 3585-3595 (2002)

18. Chae, D, Lee, J: Regularity criterion in terms of pressure for the Navier-Stokes equations. Nonlinear Anal. 46, 727-735 (2001)

19. Dong, B, Chen, Z: Regularity criteria of weak solutions to the three-dimensional micropolar flows. J. Math. Phys. 50, 103525 (2009)

20. Dong, B, Jia, Y, Chen, Z: Pressure regularity criteria of the three-dimensional micropolar fluid flows. Math. Methods Appl. Sci. 34, 595-606 (2011)

21. Fan, J, Jiang, S, Ni, G: On regularity criteria for the $n$-dimensional Navier-Stokes equations in terms of the pressure J. Differ. Equ. 244, 2963-2979 (2008)

22. Zhang, $X$, Jia, Y, Dong, BQ: On the pressure regularity criterion of the 3D Navier-Stokes equations. J. Math. Anal. Appl. $393,413-420(2012)$

23. Chen, Q, Zhang, Z: Regularity criterion via the pressure on weak solutions to the 3D Navier-Stokes equations. Proc. Am. Math. Soc. 135, 1829-1837 (2007)

24. Dong, B, Chen, Z: Regularity criterion of weak solutions to the 3D Navier-Stokes equations via two velocity components. J. Math. Anal. Appl. 338, 1-10 (2008)

25. Chen, Z, Xin, Z: Homogeneity criterion for the Navier-Stokes equations in the whole spaces. J. Math. Fluid Mech. 3 $152-182(2001)$

26. Chen, Z, Price, WG: Morrey space techniques applied to the interior regularity problem of the Navier-Stokes equations. Nonlinearity 14, 1453-1472 (2001)

27. Chemin, JY: Perfect Incompressible Fluids. Oxford University Press, New York (1998)

28. Giga, Y: Solutions for semilinear parabolic equations in $L^{P}$ and regularity of weak solutions of the Navier-Stokes system. J. Differ. Equ. 62, 186-212 (1986) 